\title{
Aplicación de Trabajo en Equipo y Logro Individual (TELI) en alumnos del Grado de Medicina.
}

\author{
ESTHER ESCUDERO ${ }^{\text {a }}$, ISABEL SANCHEZ-VERA ${ }^{\text {b; }}$ URSULA MUÑOZ ${ }^{c}$, RIMA \\ BARHOUM $^{d}$, MARIA CRUZ SÁDABA ${ }^{\mathrm{e}}$ y ASIER JAYO ${ }^{\mathrm{f}}$ \\ a estheresc@ceu.es; $\quad{ }^{b} \underline{\text { isanver@ceu.es; }} \quad$ cursula.munozmoron@ceu.es; $\quad$ drbarho@ceu.es; \\ mariacruz.sadabaargaiz@ceu.es e; fasier.jayoandrés@ceu.es \\ Sección de Fisiología. Facultad de Medicina. Universidad CEU San Pablo, Madrid.
}

\section{Abstract}

In the first semester of the academic year 2018 2019, the professors of Physiology at the Medicine School CEU San Pablo University, have used the collaborative learning modality TELI to teach Physiology of the Blood. To develop the TELI technique, the students were divided into two groups, one of them received the traditional Master Class and the other group received the TELI teaching. The students worked in the classroom forming groups of 4 people. At the beginning of each of the three blood topics, the teacher raised different questions and the students had 15 minutes to hold a brainstorming session and share their initial knowledge. After that, the students had to make a diary of notes following the ideas of brainstorming and consulting different sources such as the material of the Blackboard platform. Finally, the evaluation of the knowledge of each student was carried out with a written individual test. When comparing the successes in the answers of the questionnaire, the groups that did the collaborative work obtained better scores than those that did not. Nevertheless, the perception from the students about this methology was not very positive.

Keywords:TELI, Cooperative work, Physiology, Medicine.

\section{Resumen}

Durante el primer semestre del actual curso académico 20182019 los profesores de Fisiología I del Grado de Medicina, han utilizado el aprendizaje colaborativo TELI en el bloque Fisiología de la Sangre. Para ello, los alumnos se distribuyeron en un grupo con el que se trabajó con TELI y otro, en el que se impartió la docencia con Clase Magistral. Para el desarrollo con TELI los alumnos trabajaron en el aula en equipos de 4 personas. Al inicio de cada uno de los tres temas de los que consta el bloque de Fisiología de la Sangre, el profesor planteó cuestiones y durante 15 minutos los alumnos, utilizando un Folio Caótico, realizaron una Lluvia de Ideas poniendo en común sus conocimientos previos sobre el tema a tratar. Después, se les proporcionó material didáctico elaborado por los profesores a través de la plataforma Blackboard. Posteriormente cada equipo, siguiendo la guía de sus Folios Caóticos, elaboró un Cuadernillo de Apuntes que fue evaluado en forma conjunta. Finalmente se evaluaron de forma individual los conocimientos utilizando un cuestionario escrito de tipo test. Comparando los aciertos en las respuestas, los grupos que hicieron el trabajo colaborativo obtuvieron mejor puntuación que los que no, pero la acogida de la metodología no ha sido muy positiva por parte de los alumnos.

Palabras clave: TELI, Trabajo Cooperativo, Fisiología, Medicina 


\section{Introducción.}

Durante los últimos años los profesores de Fisiología I del Grado de Medicina practicamos de forma activa actualizaciones en la metodología de la enseñanza. Una de las motivaciones principales que nos empuja es la creencia de que el aula es uno de los lugares donde los estudiantes desarrollan muchas de las competencias que necesitaran para afrontar el futuro. No solo competencias cognitivas, sino también emocionales. El foco de nuestra innovación estos años, ha estado puesto directamente en fomentar su autonomía para progresar en el conocimiento de la asignatura. Para ello les hemos ido ofreciendo diferentes herramientas que han tenido, en general, buena aceptación por los alumnos y que han demostrado tener efectos positivos en el aprendizaje de nuestra materia. (Escudero et al 2017; Sánchez-Vera et al 2017, Escudero et al 2018, Muñoz et al 2018).

Durante este año uno de los planteamientos que hemos abordado es fomentar el trabajo colaborativo en grupos pequeños entre los alumnos de Medicina, ya que últimamente venimos detectando, cada vez más, una tendencia por parte de este alumnado a focalizar su aprendizaje en la dirección de superar el examen MIR, que obligatoriamente tendrán que afrontar al finalizar sus estudios. Esta situación se va desplazando también hacía los cursos básicos iniciales de la titulación, lo que hace que de forma anticipada se generen perfiles individualistas. Por ello, hemos planteado utilizar en el bloque de Fisiología de la Sangre la herramienta de aprendizaje basada en pequeños grupos cooperativos, tomando como base la herramienta Trabajo en Equipo-Logro Individual (TELI) descrita por Slavin en la Universidad Johns Hopkins.

El trabajo cooperativo permite mejorar el aprendizaje mediante la creación de equipos que trabajan en alto rendimiento, de tal manera que cooperan entre ellos generando a su vez una competencia positiva para demostrar que todos los miembros del grupo terminan alcanzando el nivel de aprendizaje deseado. (Larry Michaelsen et al. 2004, Ricard, 2010., Estelles-Miguela et al 2017). Además, al recibir puntuaciones tanto por el trabajo en cooperación como por los logros individuales conseguidos como resultado del trabajo en equipo, favorece que todos los integrantes se impliquen en la actividad evitando "polizontes".

Consideramos que aunque este tipo de modelo cooperativo no debe sustituir la enseñanza del profesor, será muy positivo para fomentar las habilidades de pensar, poner en común, cotejar información, integrar y aplicar los conocimientos previos de los alumnos. Además esperamos que el hecho de que cada uno de ellos pueda mejorar de forma particular su rendimiento académico, habiéndolo conseguido después de cooperar en la generación de conocimiento junto con sus compañeros, pueda ajustarse satisfactoriamente al perfil del estudiante actual de Medicina.

\section{Objetivos.}

El objetivo principal de este trabajo es proponer una nueva metodología de aprendizaje del bloque de Fisiología de la Sangre, basada en Trabajo en Equipo cooperativo y Logro Individual (TELI).

Se trata de una serie de métodos de enseñanza en los que los alumnos trabajan en grupos pequeños para que evalúen lo que saben ellos y los demás, discutan con sus compañeros, superen los posibles problemas de comprensión de algunos conceptos, y generen conocimiento juntos. 
La finalidad última es intentar rebajar el perfil individualista que aparece cada vez más temprano en los estudiantes de Medicina, evitando los efectos negativos de la competencia para enfocarla como mecanismo para motivar a los alumnos a dar lo mejor de sí mismos.

\section{Desarrollo de la innovación.}

Presentamos el análisis y las conclusiones sobre la utilización de la metodología de aprendizaje basada en equipos cooperativos puesta en marcha en el bloque de Fisiología de la Sangre, perteneciente a la asignatura Fisiología I de la titulación de Medicina. La asignatura de Fisiología I se imparte en el primer semestre del $2^{\circ}$ curso, es de carácter obligatorio y consta de 7,5 créditos ECTS.

El número de alumnos matriculados en la asignatura en el curso 2018-2019 fue de 163, y estaban repartidos en cuatro grupos. (Tabla 1)

\begin{tabular}{cc}
\hline $\begin{array}{c}\text { Grupo de } \\
\text { teoria }\end{array}$ & $\begin{array}{c}\text { Número de } \\
\text { alumnos }\end{array}$ \\
\hline 01 & 40 \\
02 & 38 \\
03 & 35 \\
04 & 50 \\
\hline
\end{tabular}

Los alumnos de los grupos 02 y 04 (88) recibieron la docencia sobre Fisiología de la Sangre con metodología de Clase Magistral (los llamamos Grupo Clase Magistral), y los alumnos de los grupos 01 y 03 (75) utilizaron la metodología de Trabajo en Equipo-Logro Individual (los llamamos Grupo TELI).

El bloque de sangre se dividió en tres temas: 1, Introducción y eritrocitos. 2, Trombocitos. 3, Leucocitos. Para su impartición el primer tema contó con 4 días de clase, y los temas 2 y 3 con tres clases cada uno.

Los alumnos del Grupo TELI trabajaron en el aula organizados en equipos de 4 personas. No se les obligó a cumplir ningún requisito para formar parte de los equipos, de tal manera que se constituyeron aleatoriamente.

Al inicio de cada tema, cada equipo contó con un folio A3 (Folio Caótico) en el que cada uno de los 4 componentes debía escribir con un color diferente las ideas que le surgían para responder a las preguntas que iba formulando el profesor. La finalidad consiste en generar un espacio sobre papel para que cada alumno recuerde conocimientos o elabore pensamiento propio sobre una cuestión concreta del tema a modo de Lluvia de Ideas o Brainstorming. En este apartado se animó a los alumnos a que relacionaran conceptos aprendidos en otras asignaturas como Biología Celular, Histología o Bioquímica (de $1^{\mathrm{er}}$ curso) para compartir con el equipo. Los Folios Caóticos se entregaron como material evaluable. 
Con ayuda de una presentación de Power Point, el profesor, interactuando como mediador y facilitador de conocimiento, lanzaba cuestiones apoyadas en imágenes o esquemas. Las primeras preguntas iban destinadas a que los alumnos bucearan en su conocimiento previo como base desde donde continuar el estudio del tema. La intención era que cada alumno y cada equipo evaluase su punto de partida respecto al tema propuesto. Las preguntas posteriores estaban orientadas a plantear los objetivos que debían conseguir los alumnos para dominar los conceptos nuevos que aprender.

Una vez hechas las preguntas se les dejaba generar una Lluvia de Ideas durante 15 minutos.

Posteriormente con la ayuda de material didáctico subido a la plataforma BlackBoard por el profesor, cada equipo tuvo que elaborar un Cuadernillo de Apuntes del bloque siguiendo las ideas plasmadas en sus Folios Caóticos. Para terminarlo, después de los días de clase los alumnos contaron con 2 días más de trabajo fuera del aula antes de la entrega.

Finalmente se les hizo un cuestionario individual de tipo test con preguntas multiopción y una única respuesta correcta, el cual fue el mismo para los alumnos del Grupo Clase Magistral.

Para los alumnos del Grupo TELI, se evaluó de forma conjunta los tres Folios Caóticos y el Cuadernillo de Apuntes y de forma individual el cuestionario de tipo test.

\section{Resultados.}

Para analizar los resultados obtenidos en el rendimiento de los alumnos sobre la compresión de los fundamentos fisiológicos que rigen la sangre, llamamos Grupo Clase Magistral a los 88 alumnos que no participaron en la innovación docente y denominamos Grupo TELI a los 75 alumnos que trabajaron a través de equipos.

\section{Análisis de las calificaciones obtenidas por cada alumno en el test individual.}

\subsection{Porcentaje de aprobados en cada grupo.}

Hemos analizados el porcentaje de alumnos capaz o no de superar la prueba escrita de conocimientos mínimos exigidos sobre el tema de Fisiología de la Sangre. Para la prueba se diseñó una batería de preguntas de tipo test en los que había 4 opciones de las cuales únicamente una era correcta.

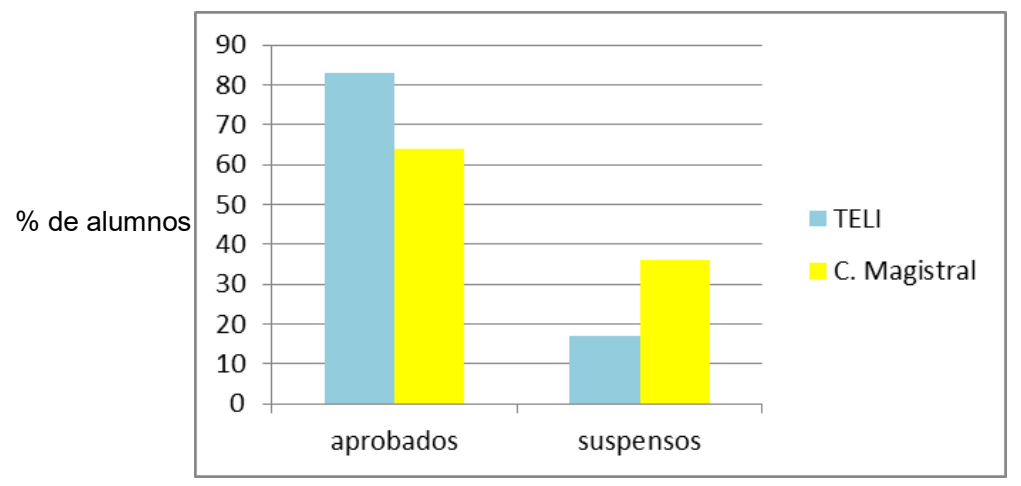

Figura 1. Se representa el porcentaje de alumnos aprobados. En azul los alumnos que hicieron la innovación (Grupo TELI) y en amarillo los que recibieron la metodología tradicional (Grupo Clase Magistral). 
Como se aprecia en la gráfica de la figura 1, el $83 \%$ de los alumnos pertenecientes al grupo TELI superaron la prueba mientras que el grupo de Clase Magistral lo hicieron el 64 \%. El porcentaje de suspensos fue prácticamente el doble en el grupo de alumnos que recibieron la enseñanza con el método de la clase magistral en comparación con el grupo TELI (36 \% vs $17 \%)$.

\subsection{Calificación media obtenida en cada grupo.}

Como se observa en la figura 2, la calificación media obtenida en el grupo de la Clase Magistral fue de 5.3 y al comparar con el grupo TELI se observa una mejora de 1.2 puntos en la media de estos alumnos que obtuvieron una calificación media de 6.5 puntos.

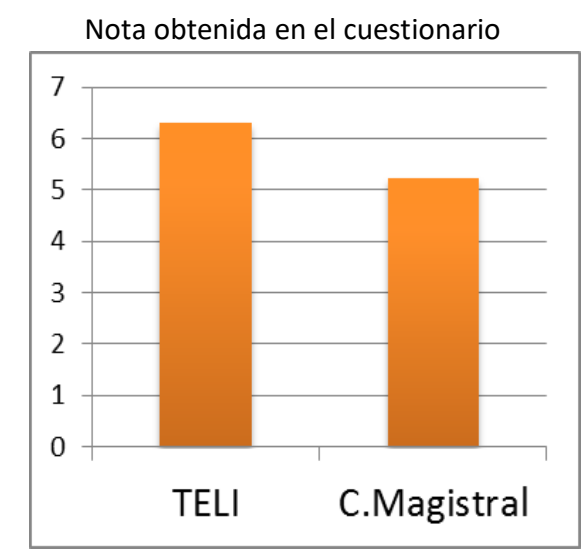

Figura 2. Se representa la nota obtenida en el cuestionario.

\subsection{Cuantificación del número de preguntas acertadas en cada grupo.}

Analizamos el porcentaje de alumnos que conseguían contestar más preguntas correctamente.

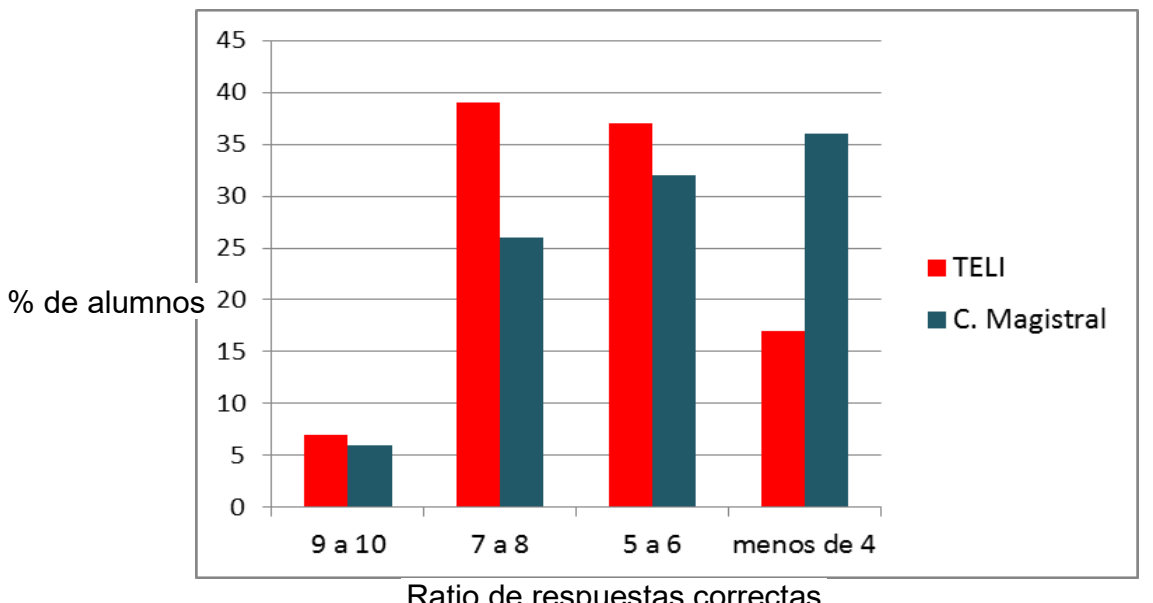

$\underline{\text { Ratio de respuestas correctas }}$

Figura 3. Se representa en el eje de las coordenadas el ratio de preguntas acertadas en el test. En el eje de ordenadas el porcentaje de alumnos. En rojo se representa al Grupo TELI y en azul al Grupo Clase Magistral 
En la figura 3, podemos observar que en el grupo donde se realizó la innovación docente (TELI), un 7\% de los alumnos contestaba correctamente a casi la totalidad de las preguntas (de 9 a 10 aciertos), siendo este resultado similar al obtenido en el Grupo de Clase Magistral, con un $6 \%$ de alumnos que también conseguían este ratio. Se cuantificó también, que un 39\% de los alumnos del Grupo TELI contestaron correctamente entre 7 y 8 cuestiones frente al 26\% del Grupo Clase Magistral. En el ratio de 5 a 6 preguntas correctas encontramos menores diferencias entre los porcentajes de ambos grupos, siendo de un $37 \%$ en el Grupo TELI frente a un 32\% en el Grupo de Clase Magistral. Pero donde aparecen las diferencias más significativas es en el número de alumnos que contestaron correctamente 4 o menos preguntas, en donde el Grupo TELI tiene un $17 \%$ de los alumnos frente a más del doble que aparece en el Grupo Clase Magistral (17\% vs 36\%).

\section{Análisis en el grupo TELI de las correlación entre la puntuación obtenida en la innovación y la nota obtenida en el cuestionario de tipo test.}

Quisimos conocer si dentro del Grupo TELI, existía relación entre la calificación obtenida en la valoración de la innovación (es decir la suma entre la nota de los Folios Caóticos y el Cuadernillo de Apuntes) y un mayor éxito a la hora de acertar las preguntas del cuestionario de tipo test.

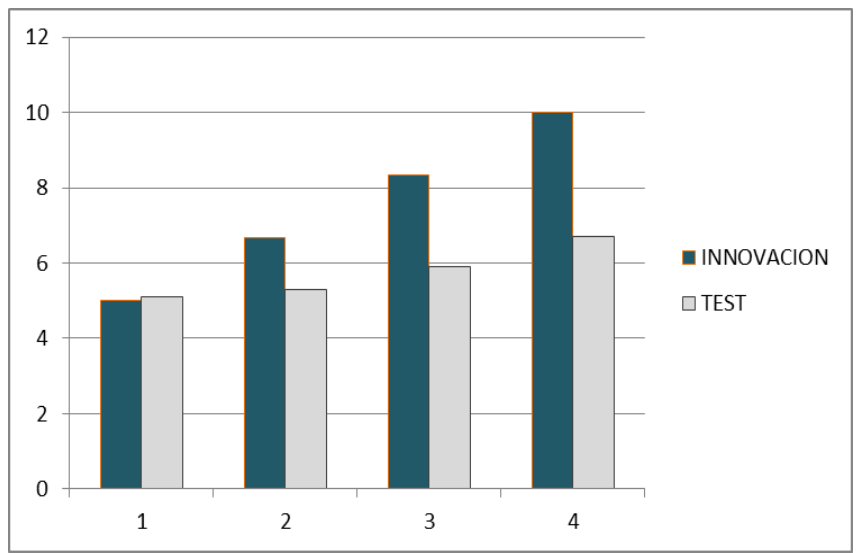

Figura 4. Se representa la relación entre la calificaión obtenida en la innovación (Folio Caótico más Cuadernillo de apuntes) y la nota del cuestionario tipo test.

Como se evidencia en la figura 4, aquellos alumnos que obtuvieron mejor valoración en la innovación tuvieron mejor puntuación en el test, pero hay que notar que a pesar de las mejores calificaciones en la innovación el aumento del resultado en el test no es significativamente llamativo. 


\section{Resultados del análisis de la encuesta de satisfacción de los alumnos con la metodología TELI.}

Para conocer el grado de satisfacción de los alumnos a la hora de estudiar el bloque de Fisiología de la Sangre con la metodología TELI, hicimos unas encuestas anónimas solo a los alumnos de este grupo.

Las preguntas que hicimos fueron las siguientes:

1 ¿Te ha resultado interesante realizar esta metodología?

2. ¿Te ha servido para mejorar tu aprendizaje del bloque de sangre?

3. ¿Te ha motivado trabajar en colaboración con tus compañeros de grupo?

4. ¿Recomendarías que siguiéramos haciendo esta actividad el próximo curso?

5. ¿En general, como valorarías tu grado de satisfacción con esta actividad?

3.1. Análisis sobre el interés por la actividad de innovación, la mejora del aprendizaje y la motivación para trabajar en equipo.

En la tabla 2 se recogen las valoraciones de los alumnos respecto a las tres primeras cuestiones que se les formularon en la encuesta de satisfacción de la actividad.

\begin{tabular}{|c|c|c|c|c|}
\hline & \multicolumn{2}{|c|}{ FOLIO CAÓTICO } & \multicolumn{2}{|c|}{ CUADERNILLO APUNTES } \\
\hline & SI & NO & SI & NO \\
\hline INTERES & $40 \%$ & $60 \%$ & $51 \%$ & $49 \%$ \\
\hline FACILIDAD ESTUDIO & $36 \%$ & $64 \%$ & $37 \%$ & $63 \%$ \\
\hline TRABAJO EN EQUIPO & $54 \%$ & $46 \%$ & $59 \%$ & $41 \%$ \\
\hline
\end{tabular}

Como se aprecia en la tabla 2, despertó más interés en los alumnos la realización del Cuadernillo de Apuntes que la participación en la Lluvia de Ideas utilizando el Folio Caótico ( $51 \%$ vs $40 \%$ ). El trabajo en equipo ha sido valorado positivamente por más de la mitad de la clase tanto en con los Folios Caóticos como en la elaboración del Cuadernillo de Apuntes (54\% vs 59\%). Sin embargo, cuando les preguntamos sobre si consideraban que realizar estas actividades les habia facilitado el aprendizaje, un 64\% opinó que el Folio Caótico no les habia ayudado a estudiar y el $63 \%$ opinó que hacer los apuntes tampoco.

3.2. Análisis sobre el grado de satisfacción con las actividades y si recomendarían su realización.

En las figuras 5A y 5B se representa el porcentaje de alumnos que expresaron su grado de satisfacción con las actividades de Folio Caótico y elaboración del Cuadernillo de Apuntes. 
Se observa que hay más aceptación con la propuesta de elaborar apuntes que la de generar Brainstorming utilizando el Folio Caótico. Si se valora en conjunto los alumnos que consideraron ligeramente o muy satisfactorio su realización, sólo una media del $40 \%$ de los alumnos refirieron estar satisfechos con la innovación.

\section{Cuadernillo Apuntes}

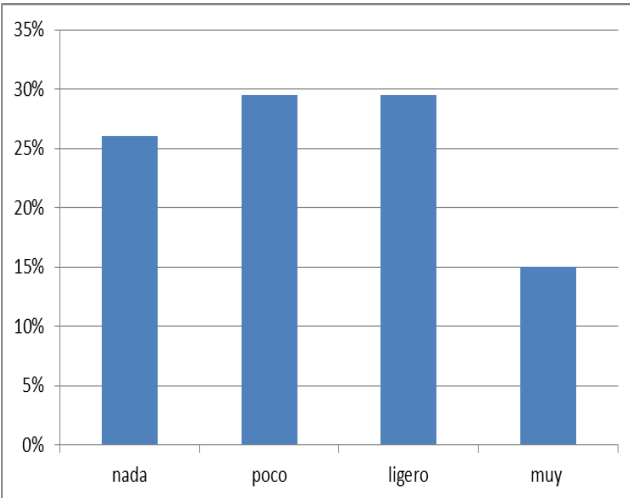

Folio Caótico

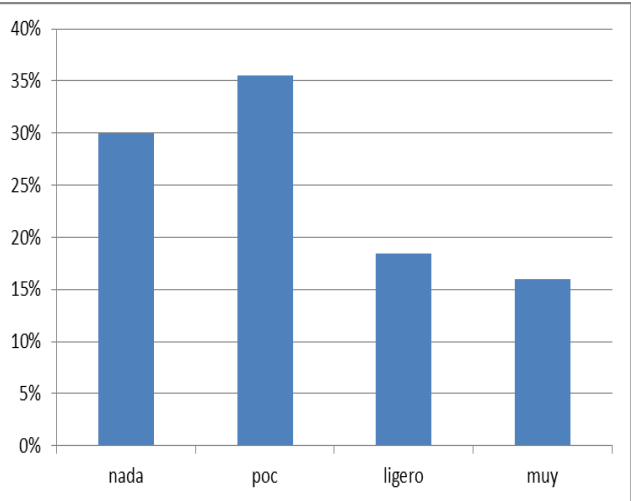

Figura 5. Se representa la valoración de los alumnos respecto al grado de satisfacción con la elaboración del cuadernillo (Fig. 5A) y la participación en el Folio Caótico (Fig. 5B).En ambas gráficas en el eje de ordenadas se expresa el porcentaje de alumnos y en el de coordenadas los distintos grados de satisfacción

Por último, en la figura 6 se representa si los alumnos recomendarían hacer estas actividades en próximos cursos, pudiendo observar que en ambos casos (tanto en la elaboración de apuntes como en la participación del Brainstorming con el Folio Caótico) el $35.5 \%$ de los alumnos si las recomendaría frente al $64.5 \%$ que opina que no lo haría.

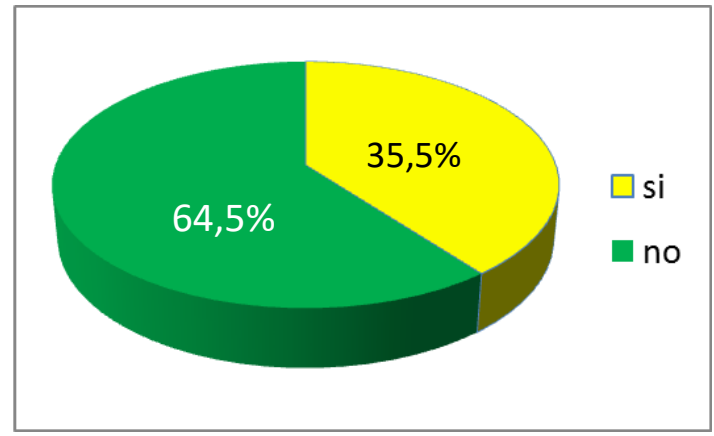

Figura 6. Se representa la valoración de los alumnos sobre si recomendarían que continuáramos con esta metodología el curso próximo. En verde se representa el porcentaje de respuestas negativas, y en amarillo el de positivas. 


\section{Conclusiones y comentarios.}

Durante los últimos cinco años, los profesores de la asignatura de Fisiología I de la Facultad de Medicina han utilizado la metodología de "aprendizaje/facilitación del aprendizaje" con los alumnos de segundo curso. Del análisis de las diferentes propuestas hemos destacado que, en general, las nuevas metodologías tienen un grado de aceptación positivo en los alumnos para aumentar su rendimiento académico (Escudero et al 2017; Sánchez-Vera et al 2017).

Además, en los dos últimos años hemos comenzado a detectar que los alumnos de medicina comienzan a potenciar un carácter competitivo e individualista desde los primeros cursos preclínicos, situación que con anterioridad se ubicaba en los últimos años de la titulación como consecuencia comprensible del reto que supone superar el examen MIR. Es por ello que este año hemos querido bajar ese perfil individual temprano para favorecer competencias de trabajo cooperativo y enfocar al alumno a dar lo mejor de sí mismo.

La innovación se ha realizado con el bloque de Fisiología de la Sangre por ser la parte del temario de fisiología más familiar para los alumnos y de la que tienen un conocimiento previo más amplio, por lo que pensamos facilitaría su inmersión en la actividad. La finalidad de la propuesta ha sido que los estudiantes elaboraran material propio del tema en cuestión, con la intención de evitar que utilizasen únicamente como fuente de estudio unos apuntes de antiguos alumnos que circulan por la Universidad.

El análisis sobre el Trabajo Cooperativo en el que se ha valorado la elaboración de los Folios Caóticos y el Cuadernillo de Apuntes, pone de manifiesto que las calificaciones que obtuvieron los alumnos participantes fueron suficientes y no hubo ningún suspenso (figura 4, barras verdes oscuras). Además, la valoración sobre el trabajo en equipo ha sido positiva para un poco más de la mitad de los alumnos, considerando un $54 \%$ de ellos positivo trabajar en equipo para hacer los Folios Caóticos y un 59\% encontró positivo cooperar para hacer el Cuadernillo de Apuntes (tabla 2). Sin embargo y sorprendentemente, el interés de los alumnos por realizar la metodología Brainstorming fue menor que el interés por elaborar apuntes $(40 \%$ vs $51 \%)$. Por otra parte, solamente un $37 \%$ de ellos encontró que esta metodología les facilitó el aprendizaje de la sangre. Estos resultados reflejan la preferencia de estos alumnos por métodos directos que les faciliten el éxito en sus pruebas escritas, más que por aquellos que les brinden crecer en otras competencias como por ejemplo la libertad para organizar el grupo, distribuir los contenidos, discutir ideas, o relacionar conocimientos de otras asignaturas.

En cuanto al análisis del abordaje del Logro Individual, evaluando los aciertos en el ejercicio de tipo test, se observa que la actividad de innovación ha sido positiva a la hora de mejorar su puntuación en este bloque de conocimiento, ya que la nota media obtenida en la prueba es más alta en los alumnos del Grupo TELI que la de sus compañeros del Grupo Clase Magistral. (Fig. 2), y además, el número de suspensos fue menor en el Grupo TELI (Fig. 1). Estos resultados en conjunto, nos lleva a los profesores a valorar positivamente la utilización de la innovación ya que ha habido una mejoria en el aprendizaje. 
Sin embargo, la opinión general de los alumnos sobre la satisfacción de aplicar este método pone de manifiesto que un $60 \%$ lo valora de manera negativa. Una observación a tener en cuanta es que la encuesta de satisfacción se hizo a ciegas, es decir, sin que los alumnos supieran el rendiminto de la innovacion sobre la mejoria de sus notas en el bloque de sangre.

Estos resultados nos hacen reflexionar sobre el bajo perfil creativo e innovador de los estudiantes de las nuevas promociones que están llegando a las aulas de Medicina, y también sobre su focalización casi única en obtener la mejor calificación. Esta situación nos debe colocar en reenfocar la docencia en el aula, ya que no solo es importante que los alumnos consigan mejorar el rendimiento académico, sino que el aprendizaje óptimo debe enfocarse tanto a las competencias puramente conceptuales como a las relacionadas con la inteligencia emocional. Consideramos que para todos los alumnos en general, pero para los de medicina en particular, es importante focalizar su atención no solo hacia la obtención de una buena puntuación sino también en valorar el conocimiento desde la perspectiva universitaria del Saber.

Por otra parte, es importante reseñar la dificultad que supone para el docente decantarse por una metodología innovadora que prevalezca por encima de las demás. Se debe reflexionar sobre que el aprendizaje no se consigue únicamente a partir de diferentes interacciones, sino que también hay que redirigirse hacia la consolidación del conocimiento mediante el esfuerzo intelectual. Siendo importante hacer hincapié que en el entorno universitario es necesario que la metodología docente no solo se focalice en las necesidades actuales de un alumnado muy influenciado por la inmediatez de una tecnología fulminante y en constante cambio, sino que empieza a ser cada vez más prioritario que la metodología docente se adapte también a las necesidades del Profesor para evitar la pérdida de la esencia del contexto universitario.

\section{Referencias}

Escudero E, Sánchez-Vera I, Barhoum R, Muñoz U, Jayo A (2018). Análisis de la metodología Flipped learnig en el entorno de la práctica de la Fisiología Médica.

Doi: http://dx.doi.org/10.4995/INRED2018.2018.8616

Escudero E, Sánchez-Vera I, Barhoum R, Puche J E, Muñoz U, (2017). Análisis del uso de autoevaluaciones en una plataforma digital en el entorno de la Fisiología Médica.

Doi: http://dx.doi.org/10.4995/INRED2017.2017.6809

Michaelsen L, Bauman A, Fink D (2004). Team-Based Learning: A Transformative Use of Small Groups in College Teaching. Stylus Publishing (VA)

Estelles-Miguela S, José Albarracín J, Guillem M, Palmer MA Peris-Ortiz M (2017) "Desarrollo de la Competencia de Trabajo en Equipo en la Asignatura de Dirección de Producción y Operaciones". Doi: http://dx.doi.org/10.4995/INRED2017.2017.6892 
Muñoz U, Escudero E, Barhoum R, Sádaba MC, Sánchez-Vera I (2018). Generación colaborativa de conocimiento. Una experiencia de aprendizaje basada en equipos o Team Based Learning (TBL) en la práctica odontológica.

Doi: http://dx.doi.org/10.4995/INRED2018.2018.8723

Ricard M, (2010). "Educación: Promueve la cooperación, no la competitividad". http://www.matthieuricard.org/es/blog/posts/educacion-promueve-lacooperacion-no-lacompetitividad.

Sánchez-Vera I, Escudero E, Muñoz U, Borrego, M J, Barhoum R, (2017). Experiencia en la elaboración de videos didácticos por alumnos de Fisiología como parte de su proceso de aprendizaje. Doi: http://dx.doi.org/10.4995/INRED2017.2017.6819

Slavin, R. E. (1983). When does cooperative learning increase student achievement? Psychological Bulletin, 94(3), 429-445. http://dx.doi.org/10.1037/0033-2909.94.3.429 